\title{
Análisis de las barreras al compromiso con la mejora continua en el servicio posventa de la industria automotriz
}

Analysis of the Barriers to Continuous Improvement Commitment in the After-sales Service of the Automotive Industry

Análise das barreiras ao compromisso com a melhoria contínua no serviço pós-venda da indústria automotive

\author{
Ricardo Romero-Zárate \\ Universidad Andina Simón Bolívar (UASB). Quito, Ecuador \\ ricardoalex10@live.com \\ https://orcid.org/0000-0001-8053-0498
}

DOI: https://doi.org/10.32719/25506641.2020.8.2

Presentado: 24 de enero de 2020 • Revisado: 31 de enero de 2020

Aceptado: 16 de marzo de 2020

Artículo de investigación

Licencia Creative Commons

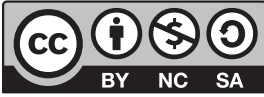




\section{Ricardo Romero-Zárate}

\section{Resumen}

El presente documento analiza las barreras al compromiso con la mejora continua (MC) en una reconocida empresa de la industria automotriz del Ecuador, con el objetivo de poder entender cuáles son las razones que aportan a esta problemática actual de este tipo de empresas, donde el personal no aporta abiertamente a cumplir los objetivos y mejorar sus procesos continuamente. La investigación fue un estudio integrado en dos centros de servicio técnico automotriz en Quito. La unidad de análisis integrada fue la población total de empleados y gerentes de una organización. Los datos se recopilaron mediante cuestionarios y entrevistas semiestructuradas. Los resultados fueron triangulados. Se utilizaron procedimientos de confiabilidad y validez para aumentar los niveles de confianza de los resultados. La investigación mostró que existen barreras en la iniciativa de los empleados para mantener un compromiso con la mejora continua y el desarrollo en las empresas, los cuales deberían superarse para implementar un sistema de calidad eficiente; además, encontró que las percepciones de compromiso de los empleados son bajas, afectando al cumplimiento efectivo de sus objetivos a corto y largo plazos. En adición, se demostró que estas barreras afectan al compromiso para lograr la adecuación y conveniencia de su sistema de calidad, lo que afecta a su cultura organizacional para lograr la mejora.

Palabras clave: Mejora continua; compromiso; gestión de calidad total; automotriz.

JEL: D21 Comportamiento de la empresa.

\section{Summary}

This document analyses the barriers to the continuous improvement commitment (MC) in a recognized company in the automotive industry in Ecuador. With the aim of being able to understand what the reasons that contribute to this current problem of this type of companies are, where the Staff does not openly contribute neither to meeting goals nor continually improving their processes, the research was an integrated study in two automotive technical service centers in Quito, Ecuador. The integrated unit of analysis was the total population of employees and managers in an organization. Data was collected through questionnaires and semi-structured interviews. The results were triangulated. Reliability and validity procedures were used to increase the confidence levels of the results. The research showed that there are barriers in the initiative of employees to maintain a commitment to continuous improvement and development in companies, which should be overcome to implement an efficient quality system; In addition, it found that the commitment of the employees are low, affecting the effective fulfilment of their short and long term objectives. It was also shown that these barriers affect the commitment to achieve the adequacy and convenience of their quality system, which directly affects their organizational culture to achieve improvement.

Keywords: Continuous improvement; commitment; total quality management; automotive. JEL: D21 Company behavior. 


\section{Resumo}

O presente documento analisa as barreiras ao compromisso com a melhoria contínua (MC) em uma reconhecida empresa da indústria automotiva do Equador com o objetivo de entender quais são as razões que contribuem para essa problemática atual nesse tipo de empresas, onde os funcionários não contribuem abertamente de forma a cumprir com os objetivos e melhorar continuamente seus processos. A pesquisa é um estudo integrado realizado em dois centros de serviço técnico automotivo em Quito, no Equador. A unidade de análise integrada foi a população total de empregados e gerentes de uma organização. Os dados foram coletados através de questionários e entrevistas semiestruturadas, e os resultados foram triangulados. Utilizaram-se procedimentos de confiabilidade e validade para aumentar os níveis de confiança dos resultados. A pesquisa mostrou que existem barreiras na iniciativa dos empregados para manter um compromisso com a melhoria contínua e com o desenvolvimento das empresas, as quais deveriam ser superadas para que se implementasse um sistema de qualidade eficiente. Além disso, concluiu-se que as percepções de compromisso dos empregados são baixas, afetando o cumprimento efetivo de seus objetivos a curto e longo prazos. Adicionalmente, demonstrou-se que tais barreiras afetam o compromisso para obter adequação e conveniência do sistema de qualidade, o que afeta a cultura organizacional para alcançar a melhoria.

Palavras-chave: Melhoria contínua; compromisso; gestão de qualidade total; automotivo. JEL: D21 Teoria da firma.

\section{Introducción y estado de la cuestión}

L

a mejora continua $(\mathrm{MC})$ es un término comúnmente utilizado en todas las industrias de diferentes sectores, para referirse al logro de tener una cultura de cambio y desarrollo de la calidad de los productos y servicios para lograr la satisfacción del cliente y reducir los costos (Saravanan y Rao 2006; Oakland 2013). El propósito para una empresa es mejorar el rendimiento con pequeños pasos para incrementar la posibilidad de ser más competitivos (Slack, Brandon-Jones y Johnston 2011). Sin embargo, la MC necesita una participación compartida de la fuerza laboral como parte de su proceso de desarrollo en las organizaciones (Oakland 2013). Por lo que, tanto el compromiso a la MC, el liderazgo de la alta dirección, así como el de los empleados deben estar vinculados entre sí para mejorar los procesos de la empresa, a fin de mantener su competitividad en el tiempo. Sin embargo, existen algunas barreras para lograr un proceso planificado, organizado y sistemático para la MC (García-Sabater, Marín-García y Perello-Marín 2012), 
que deben analizarse para minimizar sus implicaciones para luego alcanzar la excelencia.

Para lograr un enfoque eficaz de la MC, y adaptarse al cambio continuo de las necesidades del cliente, el sistema requiere la participación de toda la organización (Talib, Rahman y Qureshi 2011; Goetsch y Davis 2016). La industria automotriz no podría ser la excepción. Tanco, Mateo, Santos, Jaca y Viles (2012) explican que la MC para empresas automotrices debe ser incremental y participativa; esto significa que debe alentarse el compromiso de todos los miembros de la organización (Goetsch y Davis 2016). La calidad de los automóviles fabricados ha mejorado mucho en las últimas décadas (Lin y Lu 2006; Tanco, Mateo, Santos, Jaca y Viles 2012); sin embargo, la calidad proporcionada por los concesionarios todavía es cuestionada (Fraser, Chihiro Watanabe y Hvolby 2013). Los centros de servicio de una empresa automotriz de tipo mediana (750 empleados) en Ecuador (AEADE 2018) se han esforzado por la implementación de un sistema de mejoramiento continuo a través de un conjunto de estándares de calidad desarrollados por el fabricante de origen asiático en el exterior.

La empresa automotriz ecuatoriana logró por primera vez la certificación con los estándares de marca en septiembre de 2012, emitida por el propio fabricante. Esto permitió a la compañía lograr un progreso significativo con respecto al ahorro en costos, tiempo y mejoras de procesos (Slack 2016). Así también, se crearon nuevas responsabilidades para todo el personal, como el cumplimiento de procedimientos formales y la presencia de círculos de calidad, para lograr niveles esperados de satisfacción del cliente, así como participar activamente en la gestión del sistema (Dale, Wiele e Iwaarden 2007). Por otro lado, estos planes de acción rara vez cumplían con las fechas de implementación prometidas, los procesos no lograron los resultados esperados y el nivel de satisfacción del cliente interno aún no es el óptimo. Un estudio de García, Rivera e Iniesta (2013) demostró que podría haber poco éxito en la implementación de la filosofía de mejoramiento continuo, principalmente debido a la resistencia organizativa al cambio y la falta de una implementación adecuada y técnicas de monitoreo para la mejora. Por lo tanto, es importante investigar cuáles son las razones para entender por qué la $\mathrm{MC}$ no es un enfoque principal de gestión para los empleados, y cómo puede impactar esto tanto en el desempeño de la organización como en su 
cultura (Dale et al. 2007; Evans 2014). El objetivo es realizar un análisis de posibles barreras al compromiso -identificadas en estudios previos- para verificar si en esta organización automotriz pueden aplicarse dichas barreras, con el fin de determinar cuáles afectan directamente al compromiso actual de la misma en la MC.

\section{Revisión de literatura}

Investigaciones previas como la de García-Sabater et al. (2012) analizaron las barreras para la mejora continua en el sector automotriz. Bessant, Caffyn y Gallagher (2001) sugirió seis niveles de evolución para la MC, que son: 1 . no actividad; 2 . probar las ideas; 3 . MC estructurado y sistemático; 4. MC estratégico; 5. innovación autónoma; y, 6. la organización del aprendizaje utilizando este modelo. García-Sabater et al. (2012) descubrieron que, dependiendo de cada nivel de evolución de la MC, existen diferentes barreras que deben tratarse para fomentar la MC dentro del personal. Estas fueron la falta de capacitación en las prácticas de la $\mathrm{MC}$, la falta de implementación de las iniciativas o ideas de los empleados y la falta de un líder eficiente. Hallazgos similares en el sector automotriz fueron encontrados por García-Sabater y Marín-García (2009), enfatizando barreras como la falta de clarificación de las jerarquías y la ausencia de funciones y metodologías para desarrollar la MC.

Supuesto (S) 1: La falta de implementación de las ideas de los empleados podría ser una barrera para la MC.

Supuesto 2: La falta de promoción y reconocimiento podría ser una barrera para la $\mathrm{MC}$.

Según Singh y Singh (2015), la gestión de la MC podría ser un desafío, ya que implica cambios organizacionales. La MC significa cambio continuo, y la resistencia al cambio es un comportamiento organizacional normal (Goetsch y Davis 2016). Por ello, ambos estudios (García-Sabater y Marín-García 2009; García-Sabater et al. 2012) centran sus esfuerzos en identificar los factores críticos que deben gestionarse para implementar o mantener la $\mathrm{MC}$ en el sector automotriz, entre ellos, que se deben enfocar esfuerzos para alentar el compromiso de los empleados. Por lo tanto, se necesitan más 
exámenes para encontrar barreras específicas con respecto a fomentar la $\mathrm{MC}$ en la organización (Saravanan y Rao 2006).

Supuesto 3: Las organizaciones deben centrar sus esfuerzos en alentar el compromiso de los empleados.

Además, ambas investigaciones (García-Sabater y Marín-García 2009; García-Sabater et al. 2012) vincularon los cinco niveles de evolución de la MC de Bessant. Para algunos expertos como Wu y Chen (2006), este concepto carece de una explicación acerca de cómo las empresas pueden entender en qué etapa se encuentran actualmente, por lo que factores como la falta de liderazgo y uso inadecuado de mecanismos para alentar la MC pueden no ser precisos para gestionar las barreras y establecer aportes regenerativos para sostenerla (Yadav 2015).

Supuesto 4: La falta de liderazgo afecta la motivación de los empleados.

Supuesto 5: Uso inadecuado de herramientas y metodologías.

Torres y Gati (2011) llevaron a cabo una investigación sobre las barreras de la $\mathrm{MC}$ en el contexto latinoamericano. Se encontraron factores como la resistencia de mandos medios y la falta de liderazgo como inhibidores a la MC. Se encontró además que existe información documentada excesiva que puede afectar al cumplimiento de los criterios del sistema de MC (Assarlind y Gremyr 2016). La gerencia, en este caso de investigación, solo tiene la prioridad de lograr la certificación, en lugar de asegurarse de que este resultado se refleje en el logro de objetivos, satisfacción de las personas y su disposición a colaborar con el desarrollo de una cultura de $\mathrm{MC}$, incluyendo los métodos adecuados para dicho desarrollo (Maarof y Mahmud 2016). Evans (2014) apoya este argumento y explica que uno de los impulsores de la participación en la fuerza laboral es que los gerentes demuestren atención, preocupación y fomenten el compromiso con los objetivos de la organización.

Supuesto 6: La falta de rutas de comunicación socava el compromiso de los empleados.

Supuesto 7: Los procedimientos burocráticos afectan el compromiso de los empleados.

En general, las investigaciones analizadas (Torres y Gati 2011; García-Sabater y Marín-García 2009; García-Sabater et al. 2012 y Wu y Chen 2006) reflejaron estudios para identificar y analizar las barreras para la participación de la $\mathrm{MC}$ en el sector automotriz. 


\section{Material y métodos}

Como se sugiere en la cebolla de la investigación (Saunders et al. 2016), primero se debe considerar una filosofía de investigación para respaldar la estrategia y los métodos que se utilizarán (Saunders et al. 2016; Wilson 2014). El pragmatismo apoya diferentes posiciones filosóficas, también se enfoca en el qué y el cómo del problema de investigación (Wilson 2014), permitiendo al investigador usar los métodos considerados apropiados para generar las ideas más significativas (Wilson 2014). Por esas razones, el pragmatismo fue el enfoque filosófico elegido para la presente investigación.

El enfoque seleccionado para la recopilación e interpretación de datos fue el enfoque deductivo. Como lo señalaron Saunders et al. (2016), este ayuda a comprender, sin una metodología de investigación rígida, explicaciones alternativas de lo que está sucediendo en una situación particular. Se utilizó, además, una elección de métodos mixtos totalmente integrados de investigación cualitativa y cuantitativa para responder al objetivo de la investigación (Saunders et al. 2016). Los métodos cualitativos se utilizaron en forma de entrevistas, mientras que los métodos cuantitativos se utilizaron para los cuestionarios. El diseño de triangulación concurrente se utilizó para interpretar ambos resultados juntos (entrevistas y cuestionarios) para proporcionar una respuesta más rica y completa a esta investigación (Saunders et al. 2016).

La naturaleza de la investigación identificada fue un estudio exploratorio, ya que ayuda a aclarar la comprensión de un problema en particular, donde actualmente no existe ningún trabajo al que referirse (Saunders et al. 2016; Wilson 2014).

La estrategia de investigación fue un estudio de caso integrado, para obtener múltiples perspectivas para un análisis detallado e intensivo de una sola organización o de un caso (Cooper y Schindler 2014), basado en subunidades de análisis, lo que significa revisar la información desde la perspectiva de los mandos operativos, los mandos medios y de la alta dirección (Saunders et al. 2016; Bryman y Bell 2011; Wilson 2014), que en este caso fueron la alta gerencia, la gerencia media y operarios de línea, para entender cómo los supuestos se evidenciaban o no. 
El horizonte temporal permite definir la línea de tiempo de la investigación. Se ha elegido un horizonte de enfoque transversal, cuyo propósito ayuda a estudiar las barreras para la participación de los empleados para la MC en un momento particular (Saunders et al. 2016; Wilson 2014), en lugar de realizar entrevistas y cuestionarios durante un período prolongado (Bryman y Bell 2011).

El censo fue el método utilizado, ya que se pudo recopilar y analizar todos los datos de cada caso posible (Saunders et al. 2016). Wilson (2014, 210) afirma que la población es un "grupo claramente definido de sujetos de investigación que se está muestreando". Saunders et al. (2016) sugieren que, para una población de menos de 50 casos, es importante recuperar información de toda la población. Por lo tanto, la muestra fue toda la población de cuarenta y cuatro miembros del personal. La alta dirección (gerencia de servicio) y mandos medios (jefaturas de talleres) (2) fueron entrevistados individualmente. Se le pidió al resto del personal (42 empleados) que completaran un cuestionario para entender las razones de la falta de compromiso con la MC.

La investigación utilizó entrevistas semiestructuradas, para obtener apertura en las respuestas del entrevistado (Saunders et al. 2016; Wilson 2014). Sin embargo, Wilson (2014) argumenta que el entrevistado puede sentirse incómodo al ser grabado y por esa razón puede dejar de divulgar información interesante y sensible. Por lo tanto, las entrevistas no se grabaron, pero las ideas de la conversación se destacaron y se escribieron en un cuaderno para no perder ninguna información importante.

Además, un cuestionario fue el método utilizado para juntar la información en campo, se utilizó para recopilar datos tanto cualitativos como cuantitativos (Wilson 2014). Finalmente, según lo sugerido por Cooper y Schindler (2019), se utilizó una escala no comparativa para evaluar el grado de percepción Likert, que consiste en 5 niveles ( 1 al 5 , siendo 1 el más bajo puntaje y 5 el más alto) para desarrollar las preguntas del cuestionario, con el propósito de expresar una actitud favorable o desfavorable hacia el objeto de interés. Además, se utilizó una escala de lista de clasificación múltiple para proporcionar una medida relativa (clasificación) de las posibles barreras para la MC dentro de la organización (Cooper y Schindler 2019). 


\section{Análisis de datos}

Se utilizó el método de análisis de datos exploratorios (EDA) para usar diagramas y comparaciones de los datos, lo que permitió flexibilidad para introducir análisis no planificados (Turquía 1977 citado en Saunders et al. 2016). Se utilizó Microsoft Excel para procesar datos cuantitativos recuperados de los cuestionarios, con el fin de comparar tendencias a través de gráficos de barras (Saunders et al. 2016). Las preguntas de las entrevistas y cuestionarios abordaron cada uno de los supuestos. El investigador utilizó gráficos de barras, ya que permitieron mostrar los valores más bajos y altos de una manera fácil para apoyar una comprensión clara de la información dada (Oakland 2007).

Para la información cualitativa de los cuestionarios, se seleccionó una inducción analítica con el fin de tener un examen en profundidad de las barreras al compromiso (Saunders et al. 2016). La información se transcribió a Microsoft Excel, luego se analizó y trianguló con la información relevante de las entrevistas. Para elegir si la información era relevante o no, se argumentó en la línea de Cooper y Schindler (2014), quienes dicen que la información del entrevistado debe articularse verbalmente, lo que significa analizarse textualmente, para garantizar la riqueza del detalle deseado. Así fue como se seleccionó la información relevante de las entrevistas, para, en un segundo momento, vincular las ideas a las propuestas y su posterior análisis.

\section{Limitaciones}

Como la investigación se desarrolló únicamente en una organización del sector automotriz, y para comprender un fenómeno específico que ocurre dentro del alcance de la investigación, no hubo implicaciones externas como políticas ambientales o legales. No obstante, se detectaron obstáculos; o, la principal limitación fue la generalización, que dificultó la replicación de la investigación en organizaciones similares con un gran número de población. En segundo lugar, dado que los encuestados eran el personal administrativo, podría haber una tendencia a ser parcial en sus respuestas, ya que querrían causar una buena impresión de/para su administración. Además, los encues- 
tados podrían haber sentido que estaban obligados a completar el cuestionario, lo que podría haber disminuido la calidad de sus respuestas. Finalmente, otra limitación fue que las respuestas a los problemas podrían estar sesgadas si los entrevistados estaban extremadamente ocupados o de malhumor (Sekaran y Bougie 2013).

\section{Análisis y resultados}

Se respondieron un total de 41 cuestionarios de 44 , logrando un total de $92 \%$ de tasa de respuesta. Por otro lado, las dos entrevistas se llevaron a cabo tanto para la alta gerencia como para los representantes de la gerencia media. Para Baruch y Holtom (2008, citado en Saunders et al. 2016), las tasas de respuesta de aproximadamente $50 \%$ y 35 a $40 \%$, respectivamente, son razonables. Sin embargo, a Neuman (2005 citado en Saunders et al. 2016) sugiere un $90 \%$ para entrevistas cara a cara. Esta investigación logró el 100\% de las entrevistas cara a cara. Por lo tanto, los resultados son representativos.

Para los cuestionarios se utilizó un método de dicotomía múltiple para la codificación, utilizando una variable separada para cada respuesta diferente (Saunders et al. 2016). Las preguntas se clasificaron en cinco categorías: totalmente en desacuerdo, en desacuerdo, neutrales, de acuerdo y totalmente de acuerdo, con una codificación de 0 a 5 , respectivamente. Se utilizaron los gráficos de barras múltiples, que es la forma más práctica de comparar valores y categorías (Saunders et al. 2016). Por lo tanto, este método de comparación permitió buscar información específica de las ideas y parámetros motivadores del empleado de una manera visual y separada, lo que redujo el tiempo considerablemente durante el análisis.

Análisis de las entrevistas: se realizaron dos entrevistas, cara a cara, semiestructuradas en diferentes fechas, al Gerente 1 (ENT01) y al Gerente 2 (ENT02) de los centros de servicio. Según lo sugerido por Saunders et al. (2012), se siguió un enfoque genérico de cinco pasos para analizar los datos cualitativos. Las entrevistas fueron categorizadas de acuerdo con los supuestos de investigación, analizadas para cualquier relación. Cada hallazgo se clasificó según una categoría apropiada o, en otras palabras, según cada supuesto. 


\section{Análisis del caso de estudio y resultados}

El S1 sugirió que la falta de implementación de las ideas de los empleados puede ser una barrera para las iniciativas de la MC. Mosadeghrad (2014) descubrió que un modelo de calidad total podría ser ineficaz si falta participación de los empleados, trabajo en equipo y apoyo a la Gerencia. Para S1, las preguntas sobre el liderazgo de su superior registró 17 respuestas como de acuerdo, que representan el $47 \%$ en general. Sin embargo, hubo un punto de vista mixto sobre si la organización que solicitaba ideas de los empleados a la MC, el $19 \%$ no estuvo de acuerdo, el $25 \%$ permaneció neutral y el $39 \%$ estuvo de acuerdo. Se encontraron resultados similares en las preguntas sobre la implementación de las ideas de los empleados: el $36 \%$ de los empleados está de acuerdo, mientras que el $33 \%$ se declaró "neutral". Esto puede deberse a los círculos de calidad (CC), donde se desarrollan ideas de mejora con el apoyo de los empleados. Rokke y Prakash (2012) sugieren que los CC no son suficientes, sino que los empleados deben participar en grupos de asesoramiento y trabajar en equipos para resolver problemas y mejorar. Por lo tanto, los empleados pueden sentir que los $\mathrm{CC}$ no propician el ambiente apropiado para compartir ideas y resolver problemas.

En la entrevista 01 (ENT01) se señaló que las personas no estaban comprometidas, y que sus ideas no estaban siendo escuchadas, ya que el personal daba ideas en los CC y estas pasaban a segundo plano, dada la problemática actual de la empresa respecto del compromiso de las personas a la $\mathrm{MC}$ y sus resoluciones a priori. La entrevista 02 (ENT02) describió que los empleados no están comprometidos con las iniciativas de la MC. ENT02 comentó que las ideas de los empleados son escuchadas e implementadas conforme se consideren que fueran adecuadas y no requieran recursos que superen los presupuestos disponibles. Adicionalmente, mencionó que no siempre se hace seguimiento a las acciones tomadas. En definitiva, se puede observar que las ideas de los trabajadores pueden no ser tomadas en cuenta si se requieren recursos adicionales o si no son correctamente entendidas; y otras no se conocen si se llegaron a implementar eficazmente. Esto puede demostrar que, a corto plazo, existe cada vez menos compromiso en la MC, hallazgos similares a los encontrados por Mosadeghard (2014).

-S1 es aceptado. 
El S2 dice que la falta de promoción y reconocimiento podría ser una barrera para la MC. La información obtenida de ENT01 muestra que no hay prácticas actuales de motivación o reconocimiento para los empleados que hicieron esfuerzos para apoyar la mejora en la organización. Además, ENT02 mostró que había oportunidades de promoción laboral, aunque dentro del nivel operativo era escasa. Los resultados de ENT02 muestran que no hay ningún tipo de reconocimiento por parte de sus jefes, y más bien hay una sanción verbal o monetaria y despidos cuando no cumplen con los objetivos. Además, ENT02 describió resultados similares, asegurando que no hay oportunidades de reconocimiento desde los niveles operativos. Además, la pregunta que trataba sobre los esfuerzos de la organización para promover y reconocer las iniciativas de los empleados mostró puntos de vista mixtos entre totalmente en "desacuerdo" (17\%), "en desacuerdo" $(28 \%)$, "neutral" $(28 \%)$ y "de acuerdo" (25\%). Este resultado puede deberse a la falta de variedad de recompensas para ambos individuos dentro de la organización, que no tienen que ser necesariamente formales o incluso financieras (Rokke y Prakash 2012).

Además, se preguntó si la falta de promoción pone en peligro la iniciativa de la MC, hubo un $64 \%$ de acuerdo. Esto puede deberse a que, para mejorar el compromiso de los empleados con la $\mathrm{MC}$, se debe desarrollar oportunidades de promoción (Brajer-Marczak 2014). Por lo tanto, los empleados mostraron un claro acuerdo de que la falta de oportunidades de promoción podría ser un factor de desmotivación. En conclusión, ENT01, ENT02 y las respuestas de los cuestionarios tuvieron hallazgos similares. Existe una falta de reconocimiento de variabilidad y flexibilidad que afecta el compromiso del empleado con la MC; además, de acuerdo con los resultados obtenidos, la falta de promoción a nivel operativo puede dañar las oportunidades de implementar un sistema de la MC efectivo.

- S2 es aceptado.

Para S3, se define que una barrera es no fomentar el compromiso de los empleados con la MC. Se mostró una visión mixta entre "en desacuerdo" (28\%), "neutral" (31\%) y "de acuerdo" (28\%). ENT01 sugirió que las personas no entienden el concepto de mejora, lo que no les permite ser creativos y participativos. ENT02 aseguró que hay una falta de liderazgo o habilidades gerenciales para inspirar a los empleados, por lo que actualmente no les 
importa la calidad de su trabajo o cómo mejorarlo. Brajer-Marczak (2014) afirma que la determinación de las condiciones que inspiran a los empleados a producir iniciativas de mejora potencial es clave para construir un sistema efectivo. Esto se debe a que, a medida que se implementan las mejoras, puede ser más difícil desarrollar nuevas ideas para mejorar los procesos. Por lo tanto, es importante la capacitación continua para desarrollar habilidades analíticas y de pensamiento conceptual. Los gerentes que aplican la capacitación a través de tácticas de influencia como la persuasión, la consulta, tienen más probabilidades de lograr el compromiso (Lam, O’Donnell y Robertson 2015). La alta dirección no comprometida puede ser la razón por la cual los empleados tienen una visión mixta sobre esta propuesta.

- S3 es aceptado.

S4 menciona que la falta de liderazgo de la alta dirección y mandos medios afecta la motivación de los empleados. Se obtuvo un $61 \%$ de acuerdo, lo que demuestra que más de la mitad de los empleados piensan que su superior es un líder del grupo, lo que los motiva. No obstante, el resto se mantuvo "neutral" (17\%) o en desacuerdo. Esto significa que la fuerza laboral puede estar percibiendo un sentido de liderazgo de su superior; sin embargo, es posible que no perciban que la alta dirección esté tomando en cuenta sus puntos de vista y opiniones (Mosadeghrad 2014); esto puede poner en peligro la implementación de TQM (Yamada, Poltronieri, Gambi y Gerolamo 2013). El cuestionario demostró que el $60 \%$ de los encuestados perciben que su superior escucha sus ideas. Con todo, esto puede significar que la fuerza laboral percibe un sentido de liderazgo y una atmósfera participativa entre la gerencia media y ellos; sin embargo, los empleados también sienten que no hay apoyo y liderazgo de la alta dirección a la hora de aplicar estas ideas.

ENT01 mostró un punto de vista diferente, mencionando que existe una misión clara y una estrategia de visión que es fomentada por el compromiso de la alta dirección, el liderazgo y las políticas de puertas abiertas para escuchar las ideas de los empleados. No obstante, ENT02 mencionó que no hay liderazgo en el nivel de alta gerencia sino de jefes. Claramente, existe una brecha entre lo que los empleados piensan sobre la alta dirección y lo que la alta dirección percibe sobre la situación actual de los empleados.

$>$ S4 es aceptado. 
Con respecto al S5, se registró el $67 \%$ entre las respuestas "de acuerdo" y "totalmente de acuerdo", seguido por el 19\% de "neutral" y el 12\% de desacuerdo sobre si el uso inadecuado de herramientas y metodologías afecta el compromiso de los empleados. ENT01 mostró una descripción de cómo se están utilizando las metodologías, mencionando que el desarrollo de planes de acción y monitoreo fomentaron un buen clima de trabajo de la MC. ENT02 demostró que, además de las metodologías utilizadas, como círculos de calidad, 5s, etc., y la selección del equipo adecuado para resolver problemas ha permitido aumentar la confianza en sus resultados, lo que fomenta el compromiso con la MC.

Es notable que existe una clara inclinación al acuerdo dentro de la fuerza laboral, lo que demuestra que la organización está utilizando las metodologías y herramientas adecuadas para implementar el sistema de calidad y, por lo tanto, las iniciativas de la MC. Sin embargo, Mosadeghrad (2014) descubrió que, si las técnicas y herramientas no se implementan sistemáticamente, los empleados pierden el sentido de interés en cualquiera que sea la técnica participativa. Esta es la razón por la que la falta de aportes regenerativos para mejorar y promover las técnicas reales en la organización podría causar desinterés de cierta manera, por lo que será necesario un autoexamen para investigar si las herramientas y metodologías son regenerativas y comprometen al personal constantemente (Wu y Chen 2006).

-S5 es aceptado.

En lo que respecta a S6, describe que la falta de rutas de comunicación puede ser una barrera para la MC. S5 y S6 (discutido anteriormente en S4) mostraron que existe una visión mixta entre los empleados, que muestra brechas considerables entre lo que la alta gerencia y los trabajadores de línea perciben sobre la $\mathrm{MC}$ y la organización. Se encontraron resultados similares en el cuestionario, donde se evaluaba si la organización pregunta por las ideas de las personas y si se están implementando, respectivamente, donde el $36 \%$ de los encuestados estaban "de acuerdo", el $33 \%$ se ubica en "neutral" y el $30 \%$ "en desacuerdo". Entonces, menos de la mitad de los encuestados está de acuerdo en que la organización está implementando las ideas de los empleados.

No obstante, ENT01 demostró que el Departamento de Recursos Humanos desarrolló metodologías, como encuestas anuales, lo que ha permitido 
al personal comunicar sus ideas para desarrollar un mejor clima de trabajo. Sin embargo, cuando la organización intenta comunicar problemas sobre el sistema de calidad, el participante de ENT01 percibió que la información nunca se explica bien y que los empleados no saben cuál es el propósito final. Mosadeghrad (2006) descubrió que una comunicación vertical cercana (de arriba hacia abajo y de abajo hacia arriba) puede afectar positivamente en la participación y, por ende, en la MC. Esta puede ser la razón por la cual los entrevistados perciben que las vías de comunicación, para expresar sus preocupaciones, no son efectivas.

-S6 es aceptado.

S7 describe que los procedimientos burocráticos afectan el compromiso de los empleados. El 39\% está de acuerdo y el $28 \%$ está totalmente de acuerdo, el $11 \%$ se mantuvo neutral y el resto no estuvo de acuerdo (11\%). Esto significa que la mayoría de los empleados percibieron procedimientos burocráticos que innecesariamente consumen el tiempo de los empleados. Por otro lado, ENT01 mencionó altos niveles de carga de trabajo como barrera para la MC, pero no específicamente burocracia. ENT02 no mencionó la burocracia relacionada como una barrera para la MC. No obstante, se encontró en Yamada, Poltronieri, Gambi y Gerolamo (2013) que los procedimientos burocráticos, durante la implementación de la MC, impactan directamente a factores humanos como la motivación, la credibilidad y la resistencia al cambio. Por lo tanto, entre los hallazgos del taller realizado se detectaron percepciones de burocracia que socavan los niveles de confianza y promueven la incertidumbre, lo que afecta al compromiso con la MC.

- S7 es aceptado.

\section{Discusión y conclusiones}

El objetivo fue examinar las barreras para la participación de los empleados para la MC. Sin embargo, desde S1 hasta S7 fueron aceptadas. Como se discutió en el segundo capítulo (García-Sabater et al. 2012; Singh y Singh 2015; Wu y Chen 2006; Torres y Gati 2011), se encontraron barreras para el compromiso con la $\mathrm{MC}$ en el sector posventa automotriz:

- Falta de implementación de las ideas de los empleados. 
- Falta de promoción y reconocimiento.

- Falta de esfuerzos de la organización para alentar a la MC.

- Falta de liderazgo.

- Uso inadecuado de herramientas y metodologías para sostener y promover la MC.

- Falta de rutas de comunicación entre superior-subordinado.

- La presencia de procedimientos burocráticos.

Estas barreras deberán superarse en las empresas del sector automotriz para garantizar un compromiso de los empleados con la MC, así como un desarrollo progresivo de la organización, en sinergia con las decisiones estratégicas y el liderazgo de la alta dirección, mandos medios y demás involucrados, garantizando de por sí que se homologuen criterios de gestión de mejora con los requerimientos del sector actual (Brajer-Marczak 2014; Assarlind y Gremyr 2016) y que se trabaje en equipo con el fin de mejorar la competitividad (García-Sabater et al. 2012; Kalak y Hudson 2016).

Se recomienda, además, realizar más investigaciones para analizar la situación real en otras industrias o de similar alcance, con respecto a los niveles de compromiso de los empleados con la MC, para poder comparar más resultados (Yin 2012). En el país, la situación real del sector es cambiante, las restricciones a las importaciones en 2011 generaron cambios en las estrategias y hoy en día el mercado ha despuntado (AEADE 2018).

También se recomiendan más investigación en otros casos de estudio para identificar otros factores que, en la alta dirección, influyen directamente en el comportamiento de los empleados, y viceversa, para determinar mejores estrategias para fomentar la participación de los empleados en la MC.

\section{Referencias}

Asociación de Empresas Automotrices del Ecuador (AEADE). 2018. Anuario 2018. Quito: Gestión Creativa.

Assarlind, Marcus, e Ida Gremyr. 2016. "Initiating Quality Management in a Small Company". The TQM Journal 28 (2): 166-179. http://dx.doi.org/10.1108/TQM-01-2014-0003. Bessant, John, Sarah Caffyn y Maeve Gallagher. 2001. "An Evolutionary Model of Continuous Improvement Behavior”. Technovation (21): 67-77. https://bit.ly/3dlNrth. 
Análisis de las barreras al compromiso con la mejora continua en el servicio posventa de la industria automotriz

Brajer-Marczak, Renata. 2014. "Employee Engagement in Continuous Improvement of Processes”. Management 1429-9321 (182): 88-103. http://doi.org/10.2478/manment-2014-0044.

Bryman, Alan, y Emma Bell. 2011. Business Research Methods. Oxford: Oxford University.

Cooper, Donald R., y Pamela Schindler. 2014. Business Research Methods. Nueva York: McGraw-Hill Education.

---. 2019. Business Research Methods. Nueva York: McGraw-Hill Education.

Dale, Barrie G., Wiele Van Der y Jos van Iwaarden. 2007. “Teams and Teamwork”. En Managing quality, editado por Barrie Dale, Wiele Van Der Jos van Iwaarden, 510-530. Malden: Blackwell Pub.

El Hussein, Mohamed, Sandra Hirst, Vince Salyers y Joseph Osuji. 2014. "Using Grounded Theory as a Method of Inquiry: Advantages and Disadvantages". Qualitative Report (1927): 1-14. https://bit.ly/2MgIDJU.

Evans, James R. 2014. Quality and Performance Excellence: Management, Organization, and Strategy. Boston: Cengage Learning.

Fraser, Kim, Watanabe Chihiro y Hans H. Hvolby. 2013. "Commitment to Service Quality in Automotive Dealerships: Results from an Australian Pilot Study". Journal of Services Research (131): 7-29: https://bit.ly/3dmsMoS.

García-Sabater, Julio J., y Juan A. Marín-García. 2009. "Facilitadores y barreras para la sostenibilidad de la mejora continua: un estudio en proveedores automotrices de la comunidad valenciana”. Intangible Capital 5 (2): 183-209. http://doi.org/10.3926/ic.2009.v5n2. S183-209.

García-Sabater, Julio, Juan Marín-García y Rosario Perello-Marín. 2012. "Is Implementation of Continuous Improvement Possible? An Evolutionary Model of Enablers and Inhibitors". Human Factors and Ergonomics in Manufacturing (222): 99-112. http://doi. org/10.1002/hfm.

García, Jorge, Denisse Rivera y Alejandro Iniesta. 2013. "Critical Success Factors for Kaizen Implementation in Manufacturing Industries in Mexico". International Journal of Advanced Manufacturing Technology 68 (4): 537-545. http://doi.org/10.1007/s00170-013-4750-2.

Goetsch, David, y Stanley Davis. 2016. Quality Management for Organizational Excellence: Introduction to Total Quality. Harlow: Pearson.

Kalak, Izidin El, y Roberth Hudson. 2016. "The Effect of Size on the Failure Probabilities of Smes: An Empirical Study on the US Market Using Discrete Hazard Model”. International Review of Financial Analysis (43): 135-145. http://dx.doi.org/10.1016/j.irfa.2015.11.009.

Lam, Marco, Mark O’Donnell y Dan Robertson. 2015. “Achieving Employee Commitment for Continuous Improvement Initiatives". International Journal of Operations and Production Management (352): 201-215. http://doi.org/10.1108/IJOPM-03-2013-0134.

Lin, Liang-Hung, y Iuan-Yuan Lu. 2006. "Product Quality as a Determinant of Product Innovation: An Empirical Analysis of the Global Automotive Industry". Total Quality Management 17 (2): 141-147. http://doi.org/10.1080/14783360500450434. 


\section{Ricardo Romero-Zárate}

Maarof, Mohd Ghazali, y Fatimah Mahmud. 2016. "A Review of Contributing Factors and Challenges in Implementing Kaizen in Small and Medium Enterprises". Procedia Economics and Finance (35): 522-531. http://dx.doi.org/10.1016/S2212-5671(16)00065-4.

Mosadeghrad, Ali Mohammad. 2014. "Why TQM Programmes Fail? A Pathology Approach". TQM Journal (262): 160-187. http://doi.org/10.1108/TQM-12-2010-0041.

---. 2014a. "Strategic Collaborative Quality Management and Employee Job Satisfaction". International Journal of Health Policy \& Management (44): 167-174. http://http://dx.doi. org/10.15171/ijhpm.2014.38.

Oakland, John. 2007. Statistical Process Control. Londres: Routledge.

---.2013. Total Quality Management and Operational Excellence: Text with Cases/John Oakland. Londres: Routledge.

Pun, Kit Fai, y Surujdaye Jaggernath-Furlonge. 2012. "Impacts of Company Size and Culture on Quality Management Practices in Manufacturing Organizations: An Empirical Study". TQM Journal (241): 83-101. http://doi.org/10.1108/17542731211191249.

Rokke, Connie, y Yadav Prakash. 2012. "Challenges and Barriers to Total Quality Management: An Overview". International Journal of Performability Engineering (86): 653-665. https://bit.ly/2zQBAou.

Saravanan, Rajendran, y Srinivasa Rao. 2006. "Development and Validation of an Instrument for Measuring Total Quality Service". Total Quality Management \& Business Excellence (176): 733-749. http://doi.org/10.1080/14783360600594487.

Saunders, Mark, Philip Lewis y Adrian Thornhill. 2016. Research Methods for Business Students. Harlow: Pearson.

Sekaran, Uma, y Roger Bougie. 2013. Research Methods for Business: A Skill-building Approach. Chichester: Wiley.

Singh, Jagdeep, y Harwinder Singh. 2015. "Continuous Improvement Philosophy-Literature Review and Directions". Benchmarking: An International Journal (221): 75-119. http:// doi.org/10.1108/BIJ-06-2012-0038.

Slack, Nigel, Alistair Brandon-Jones y Roberth Johnston. 2011. Essentials of Operations Management. Harlow: Pearson.

---. 2016. Operations Management. Harlow: Pearson.

Talib, Faisal, Zillur Rahman y Mohamed Qureshi. 2011. "Prioritizing the Practices of Total Quality Management: An Analytic Hierarchy Process Analysis for the Service Industries". Total Quality Management \& Business Excellence (2212): 1331-1351. http://doi.org/10.1 080/14783363.2011.625192.

Tanco, Martín, Ricardo Mateo, Javier Santos, Carmen Jaca y Elisabeth Viles. 2012. "On the Relationship between Continuous Improvement Programs and their Effect on Quality Defects: An Automotive Case Study". Total Quality Management \& Business Excellence 233 (4): 277-290. http://doi.org/10.1080/14783363.2011.637779.

Torres, Alvair Silveira, y Ana María Gati. 2011. "Identification of Barriers Towards Change and Proposal to Institutionalize Continuous Improvement Programs in Manufacturing 
Análisis de las barreras al compromiso con la mejora continua en el servicio posventa de la industria automotriz

Operations". Journal of Technology Management \& Innovation (62): 94-109. http://dx. doi.org/10.4067/S0718-27242011000200007.

Wilson, Jonathan. 2014. Essentials of Business Research: A Guide to Doing your Research Project. Los Ángeles: SAGE.

Wu, Chih Wei, y Chyong Lin Chen. 2006. “An Integrated Structural Model Toward Successful Continuous Improvement Activity”. Technovation 26 (5-6): 697-707. http://doi. org/10.1016/j.technovation.2005.05.002.

Yadav, Ramanand. 2015. “A Roadmap for Implementing Total Quality Management Practices in Medium Enterprises”. IUP Journal of Operations Management 14 (4): 7-23. https:// dx.doi.org/10.2139/ssrn.2325838.

Yamada, Tonani Tuane, Camila Fabricio Poltronieri, Lilian do Nascimento Gambi y Mateus Cecilio Gerolamo. 2013. "Why Does the Implementation of Quality Management Practices Fail? A Qualitative Study of Barriers in Brazilian Companies”. Procedia-Social and Behavioral Sciences (81): 366-370. https://bit.ly/2XheoJ0.

Yin, Robert. 2012. Case Study Research: Design and Methods. Los Ángeles: SAGE. 